\title{
CONCOMITANT CAPGRAS DELUSION AND REDUPLICATIVE PARAMNESIA - A CASE STUDY
}

\author{
Pedro Casimiro', Fábio Carneiro², Virgínia Henriques', Filipa Fernandes Martins' ${ }^{1}$, Jacqueline Ribeiro' \\ ${ }^{1}$ Psychiatry department of Hospital Garcia de Orta, EPE - Almada, Portugal \\ ${ }^{2}$ Neurology department of Hospital Garcia de Orta, EPE - Almada, Portugal
}

\section{Background and aims:}

The delusional misidentification syndromes (DMS) are psychopathologic phenomena in which a patient consistently misidentifies people, places, objects, or events. ${ }^{1}$ These include Capgras delusion (CD) and reduplicative paramnesia (RP).

In $\mathrm{CD}$ patients become convinced that someone close to them has been replaced by an impostor with strong physical resemblance..$^{2,3}$ These patients can consciously recognize familiar faces but cannot emotionally connect with them. ${ }^{1}$ RP, also known as environmental reduplication, is the subjective belief that a place has been duplicated, existing in at least two locations simultaneously. ${ }^{4}$

DMS were initially reported to be associated only with psychiatric diseases, but more recently, they have also been described in neurological conditions, and results from structural and neuroimaging studies provide support for an organic etiology. Patients with CS and reduplicative paramnesia having unilateral brain lesions strongly implicate the right hemisphere, usually the frontal lobe. ${ }^{1}$ Also, in many cases 2 or more misidentification syndromes are present in the same patient, which may indicate similar underlying pathophysiological mechanisms. ${ }^{1}$

\section{Objectives:}

To present a case study, supported by a non systematic review of the literature with the key-words "misidentification syndrome", "reduplicative paramnesia" and "Capgras delusion".

\section{Case report:}

- 71 year-old woman, partially autonomous in daily life activities, living alone in Setubal district, Portugal. Elementary school completed (4th grade).

- History of hypertension, diabetes and chronic depressive symptoms.

- Chronic medication: none

- Admitted after assessment of diminished self-care, worsening of depressive mood and persecutory delusional ideation.

- Further evaluation showed the patient believed her home and the hospital were duplicated and located in Spain. Also, she believed that some objects in her house were from her house in Portugal but others were replicas of the originals.

-She also had the conviction that her sons and grandsons were substituted by identical impostors 10 years ago and that she has not seen them since.

- Detailed neuropsychological examination revealed: MMSE 28/30, moderate change in abstraction and calculus and severe change in verbal conceptualization and perseveration. These findings are consistent with frontal and/or subcortical cognitive dysfunction.

- MRI scan showed Fazekas 3 microangiopathic leucoencephalopathy and bilateral subcortical microhemorrages, predominantly on the right hemisphere (see figures 1-4).

- After improvement of the depressive symptoms, the patient still exhibited delusional misidentification syndrome and impairment in self-care.

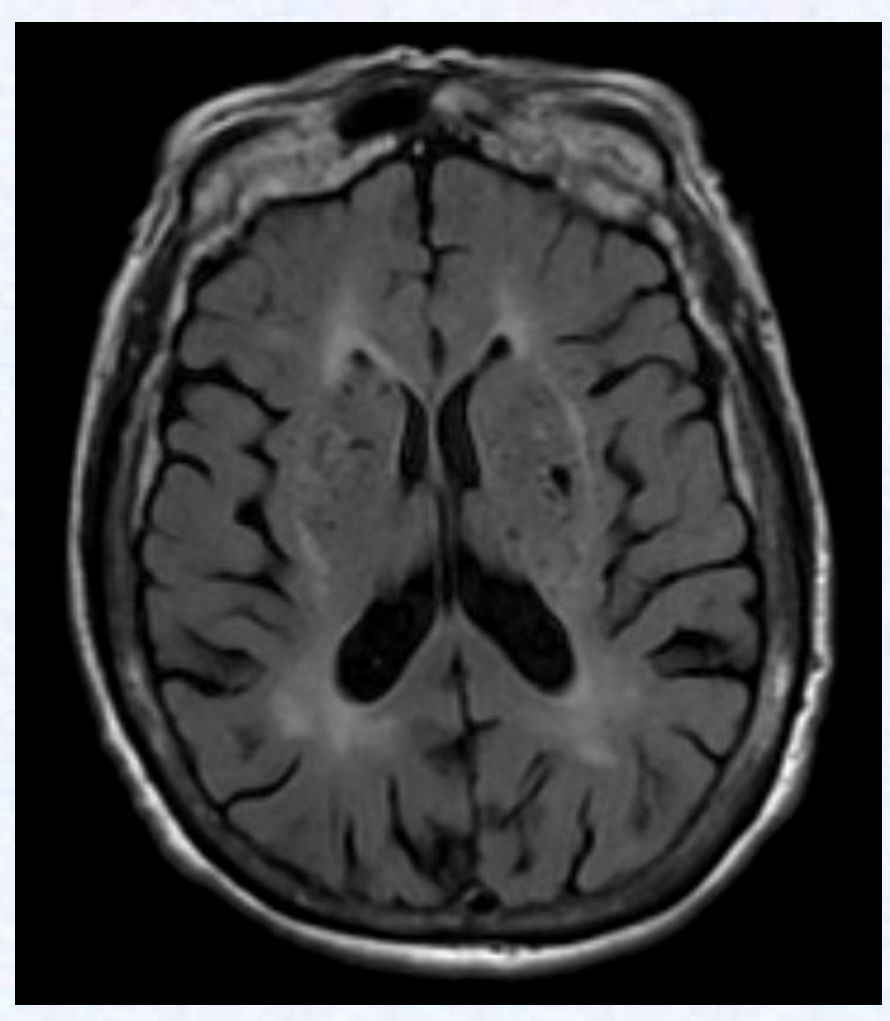

Figure 1 - Axial T2 flair

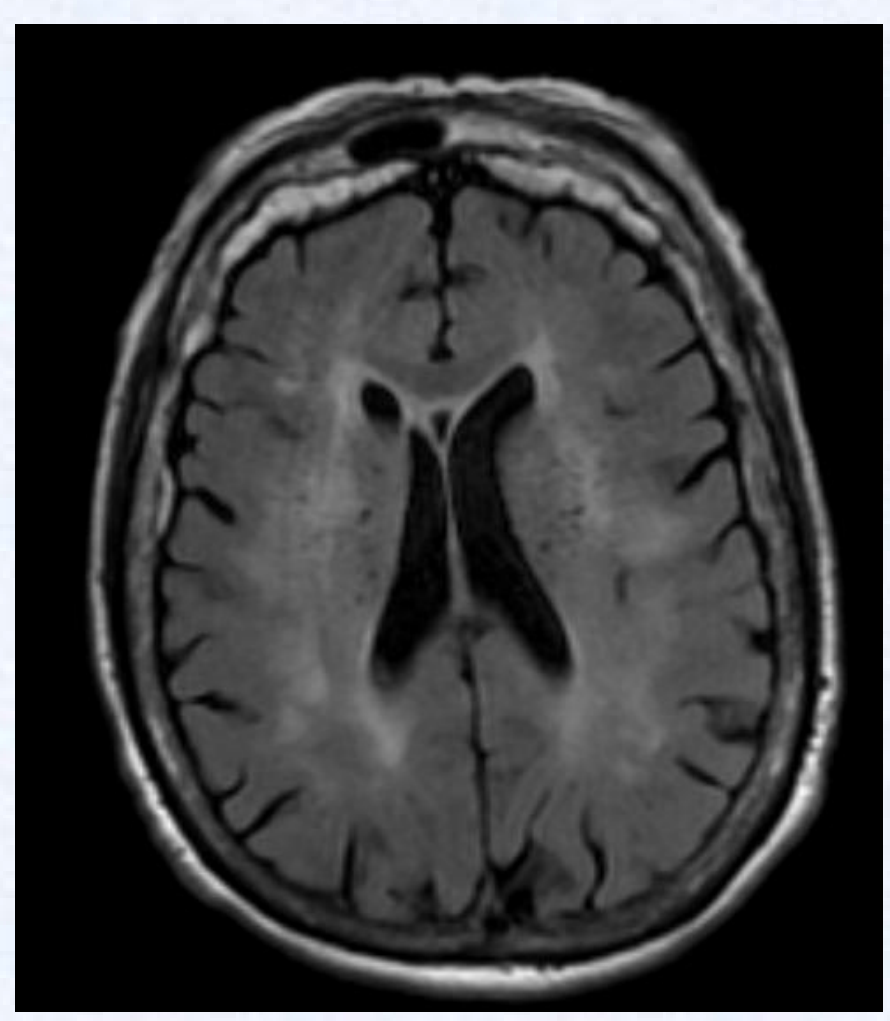

Figure 3 - Axial T2 flair

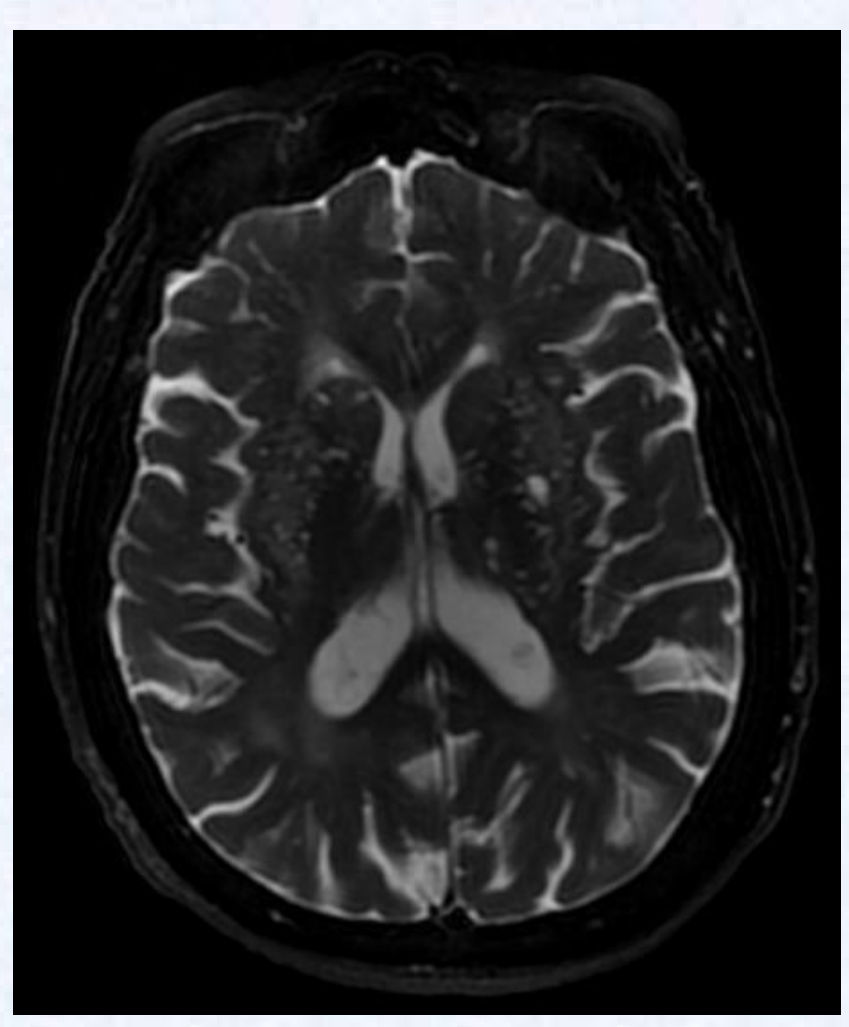

Figure 2- Axial T2

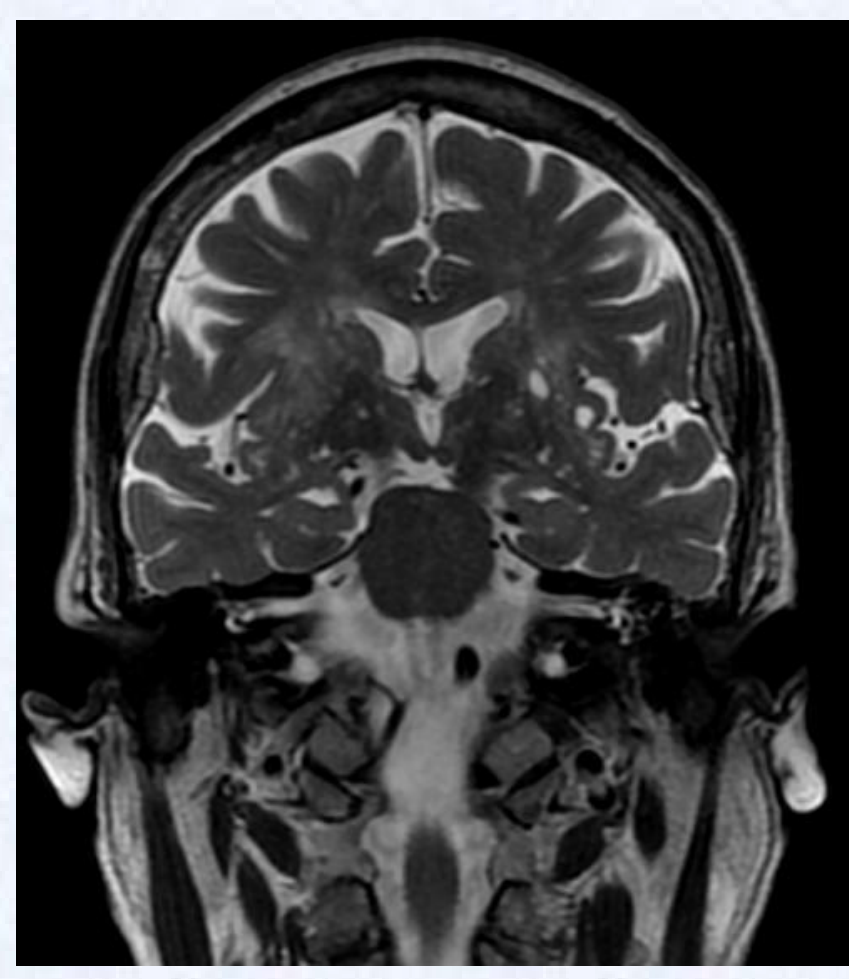

Figure 4 - Coronal T2

\section{Conclusion}

Frontal lobe dysfunction, persisting beyond resolution of depressive symptoms, and severe vascular lesions (particularly in the right hemisphere) support the hypothesis that DSM symptoms are produced, at least in part, by structural lesions. Also, the fact that this patient exhibits both CS and RP suggests similar underlying pathophysiological mechanisms for these syndromes.

DSM appears as a neuropsychiatric entity, in which cognitive, psychiatric and neuroanatomic findings converge. A multidisciplinary approach to DSM might be the key to better understand its features, cause and best course of action.

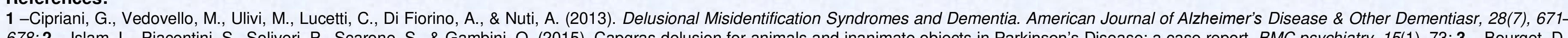

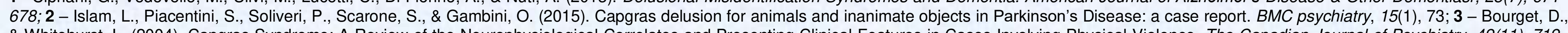

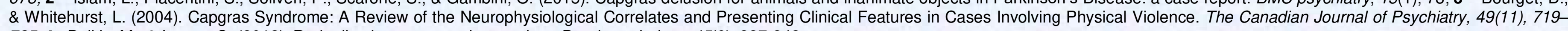
725; 4 -Politis, M., \& Loane, C. (2012). Reduplicative paramnesia: a review. Psychopathology, 45(6), 337-343. 